\title{
Design and development of topical liposomal formulations in a regulatory perspective
}

\author{
Michele Schlich ${ }^{1,4}$. Umberto M. Musazzi ${ }^{2}$. Virginia Campani ${ }^{3} \cdot$ Marco Biondi $^{3}$ - Silvia Franzé2 ${ }^{2}$ Francesco Lai ${ }^{1}$. \\ Giuseppe De Rosa ${ }^{3}$. Chiara Sinico ${ }^{1} \cdot$ Francesco Cilurzo $^{2}$ (1)
}

Accepted: 25 October 2021 / Published online: 9 November 2021

(c) Controlled Release Society 2021

\begin{abstract}
The skin is the absorption site for drug substances intended to treat loco-regional diseases, although its barrier properties limit the permeation of drug molecules. The growing knowledge of the skin structure and its physiology have supported the design of innovative nanosystems (e.g. liposomal systems) to improve the absorption of poorly skin-permeable drugs. However, despite the dozens of clinical trials started, few topically applied liposomal systems have been authorized both in the EU and the USA. Indeed, the intrinsic complexity of the topically applied liposomal systems, the higher production costs, the lack of standardized methods and the more stringent guidelines for assessing their benefit/risk balance can be seen as causes of such inefficient translation. The present work aimed to provide an overview of the physicochemical and biopharmaceutical characterization methods that can be applied to topical liposomal systems intended to be marketed as medicinal products, and the current regulatory provisions. The discussion highlights how such methodologies can be relevant for defining the critical quality attributes of the final product, and they can be usefully applied based on the phase of the life cycle of a liposomal product: to guide the formulation studies in the early stages of development, to rationally design preclinical and clinical trials, to support the pharmaceutical quality control system and to sustain post-marketing variations. The provided information can help define harmonized quality standards able to overcome the case-by-case approach currently applied by regulatory agencies in assessing the benefit/risk of the topically applied liposomal systems.
\end{abstract}

Keywords Transdermal $\cdot$ Regulatory science $\cdot$ Liposome $\cdot$ Skin penetration $\cdot$ Drug release $\cdot$ Franz cell

\section{Introduction}

The administration of drugs on the skin is as attractive as challenging. Although this route of administration allows the treatment of both local and systemic pathologies, the skin

Francesco Cilurzo

francesco.cilurzo@unimi.it

1 Dipartimento Di Scienze Della Vita E Dell'Ambiente, Sezione Scienze del Farmaco, Università Di Cagliari, via Ospedale 72, 09124 Cagliari, Italy

2 Department of Pharmaceutical Sciences, Università Degli Studi Di Milano, via G. Colombo 71, 20133 Milan, Italy

3 Dipartimento Di Farmacia, Università Degli Studi Di Napoli Federico II, via D. Montesano 49, 80131 Naples, Italy

4 Laboratory of Nanotechnology for Precision Medicine, Istituto Italiano Di Tecnologia, via Morego 30, 16163 Genoa, Italy evolved to be an excellent barrier against xenobiotics. In particular, the outermost layer of the skin, namely the stratum corneum, a multi-layered wall-like structure in which flat keratinized corneocytes are embedded in a lipophilic network, is difficultly crossed by exogenous molecules, thus limiting this route of administration to very few compounds with defined physicochemical properties. Thus, several skin penetration enhancement strategies have been investigated over the last decades. Besides the long-standing approach based on the use of chemical skin penetration enhancers, ionic liquids and physical methods for the perturbation of stratum corneum barrier [1], the use of nanotechnologies, alone or in combination with physical methods, has been investigated [2-4], and the number of publications is increasing exponentially.

The rationale behind the application of nanocarriers on the skin is the exploitation of the intercellular spaces physiologically present in the stratum corneum due to the incomplete overlapping of the corneocytes. This would ideally 
open the gap for nanocarrier penetration through the outer layer of the skin and further portioning of the cargo in the deeper layers. Nevertheless, this mechanism is not possible for many nanocarriers (such as polymeric nanoparticles that distribute mainly in the hair follicles) and questioned for others, such as lipid vesicles, for which the mechanism of penetration is not completely understood, and contradictory results are available in the literature. This could be mainly attributed to the variety of models used in the skin penetration experiments and by the experimental conditions. As an example, quantum dots (QD) were found in the liver of hairless mice after their application on the intact skin, suggesting that these nanoparticles could be absorbed after transdermal administration. However, when similar studies were carried out using rats as animal models, QD were able to reach dermis (in extent depending on their morphology and surface characteristics) [5, 6]. Similarly, QD were found to penetrate the porcine epidermis [7] and reach the stratum granulosum as in the case of fullerenes [8] but remained confined on the stratum corneum surface when applied on the human skin [9].

Besides the mechanism of penetration, many nanocarriers effectively improve the delivery of drugs in the skin, even if in some cases this is due mainly to increased solubility of the drug in the vehicle or to a better ability of the formulation to interact with SC lipids. Nanotechnologies tested on the skin move from the well-known nano and microemulsion [10] to the use of surfactant [1] or polymeric micelles [11], nanocrystals [12], solid lipid, metallic [13], polymeric nanoparticles, nanohydrogels [14] and all the family of lipid vesicles derived from liposomes [15]. Among those, the last class is, without doubt, the most studied.

Conventional liposomes were the first lipid vesicles tested for (trans)dermal delivery based on the similarity of the vesicles' composition and stratum corneum. The first evidence that conventional liposomes could be used in topical delivery of drugs dates to the work of Mezei and Gulasekharam who highlighted the penetration of entire multi-lamellar vesicles in the epidermis and dermis, which led to an increased deposition of triamcinolone acetonide with respect to conventional dosage forms [16, 17]. These results were attributed later to an experimental artefact [18] but opened the route for intensive research that culminated in the design of liposomes to be used specifically on the skin. These next generations of vesicles were developed basing on the evidence that conventional liposomes would never cross the stratum corneum as intact vesicles, disintegrating or fusing on the skin surface. On the one hand, this behaviour leads to the exchanging of lipid components with stratum corneum, thus favouring anyway the partitioning of the drug in this layer; on the other hand, it hinders the access of the drug to the deeper layers of the skin, especially in the case of a hydrophobic compound with poor affinity for the more hydrophilic epidermal layer. Then, "soft" liposomes composed of lipids with a very low transition temperature, that do not contain stabilizing agents (such as cholesterol) and containing instead destabilizing agents such as single-chain surfactants and/or ethanol have been developed. These vesicles, being more fluid, can more easily penetrate the stratum corneum barrier. In particular, they can act as drug carriers for improving the retention into the skin and the underlying tissues (e.g. transfersome ${ }^{\circledR}$ ) or enhancing the permeation through them (e.g. ethosomes) [19]. Several works have been carried out demonstrating the advantage of using these vesicles for improving the skin penetration of products with different physicochemical properties but, despite the great research effort, very few products are nowadays available on the market. Indeed, despite the dozens of clinical trials registered in dedicated US and EU sites (clinicaltrial.gov and clinicaltrialregister.eu), few medicinal products containing lipid vesicles (e.g. Pevaryl ${ }^{\circledR}$ Lipogel, Maxilene ${ }^{\circledR}$ cream, Lipoxysan ${ }^{\circledR}$, Supra-vir ${ }^{\circledR}$ cream) have been authorized as topically applied medicinal products. This can be due not only to the higher production costs but also to the more stringent and partially uncoded regulatory requirements related to the quality and safety evaluation of the final drug product, compared to conventional formulations. A nanomedicine product can be mainly designed to satisfy novel clinical requirements as well as to improve the efficacy of existing treatment, or patient compliance and adherence. In all the cases, from a regulatory point of view, the following aspects should be considered especially when the final goal is the treatment of a loco-regional pathology. First, the classical regulatory pathways for obtaining the marketing authorization cannot be applied to nanomedicines and their copies due to their intrinsic high complexity [20]. Secondly, their technological peculiarities led the regulatory authorities to consider them as non-biological complex drugs and to put in place specific and more stringent guidelines for assessing their benefit/risk balance [21]. Finally, when major post-marketing variations are required, the complexity of the formulation and the topical application limit the adoption of bioequivalence studies to assess the clinical equivalence of two products, requiring more expensive clinical trials [22].

In this work, we provide an overview of the physicochemical and in vitro biopharmaceutical characterization methods that can be applied to topical liposomal systems intended to be marketed as medicinal products, and the current regulatory provisions. Indeed, the critical quality attributes that must be defined during the pharmaceutical development depend on the general requirements of cutaneous dosage forms recently introduced by the main regulatory agencies, and the specific regulations related to nanotechnologies. 


\section{Regulatory framework}

Despite the great interest in developing nanotechnologiesand particularly liposomal formulations-to improve the bioavailability of a drug administered through the skin, no specific guidelines have been issued by the FDA and EMA for topical application, yet. However, the current regulatory framework concerning nanomedicine products, even if it is mainly focused on parenteral dosage forms, should be taken into consideration to identify and investigate the most critical quality attributes of the formulation from a physical, chemical and microbiological standpoint during the pharmaceutical development. Indeed, forced by the increased interest for biomedical applications of nanotechnology products and the difference in the biopharmaceutic pattern between nanosystems and bulk materials, the US and European regulatory framework has been updated to push manufacturers to perform more accurate pre-authorization studies for assessing the quality, safety and efficacy profile of a new nanomedicine product [21]. Both general and liposomal-specific guidelines and reflection papers have been released. For topically applied liposomal products, additional provisions can be obtained from the FDA draft guidance on nanomaterials contained in drug products [23], and the EMA guideline on coated nanomedicines [24]. Furthermore, other useful information can also be retrieved from the reflection paper released by the EMA to support manufacturers in the development of copies of intravenous liposomal products [25] or the FDA draft guidance on liposomes [26]. The requirements reported in the guidelines for the liposomal formulations and applicable also to topically applied ones are summarized in Table 1. Both agencies require a full and detailed characterization of the liposomal systems and their functionality-related excipients. In particular, the formulative studies should investigate in-depth all parameters that can influence the physicochemical quality of liposomes and their

Table 1 Additional quality critical attributes that can potentially impact on the pharmaceutical development of a liposomal formulation intended to be applied on the skin

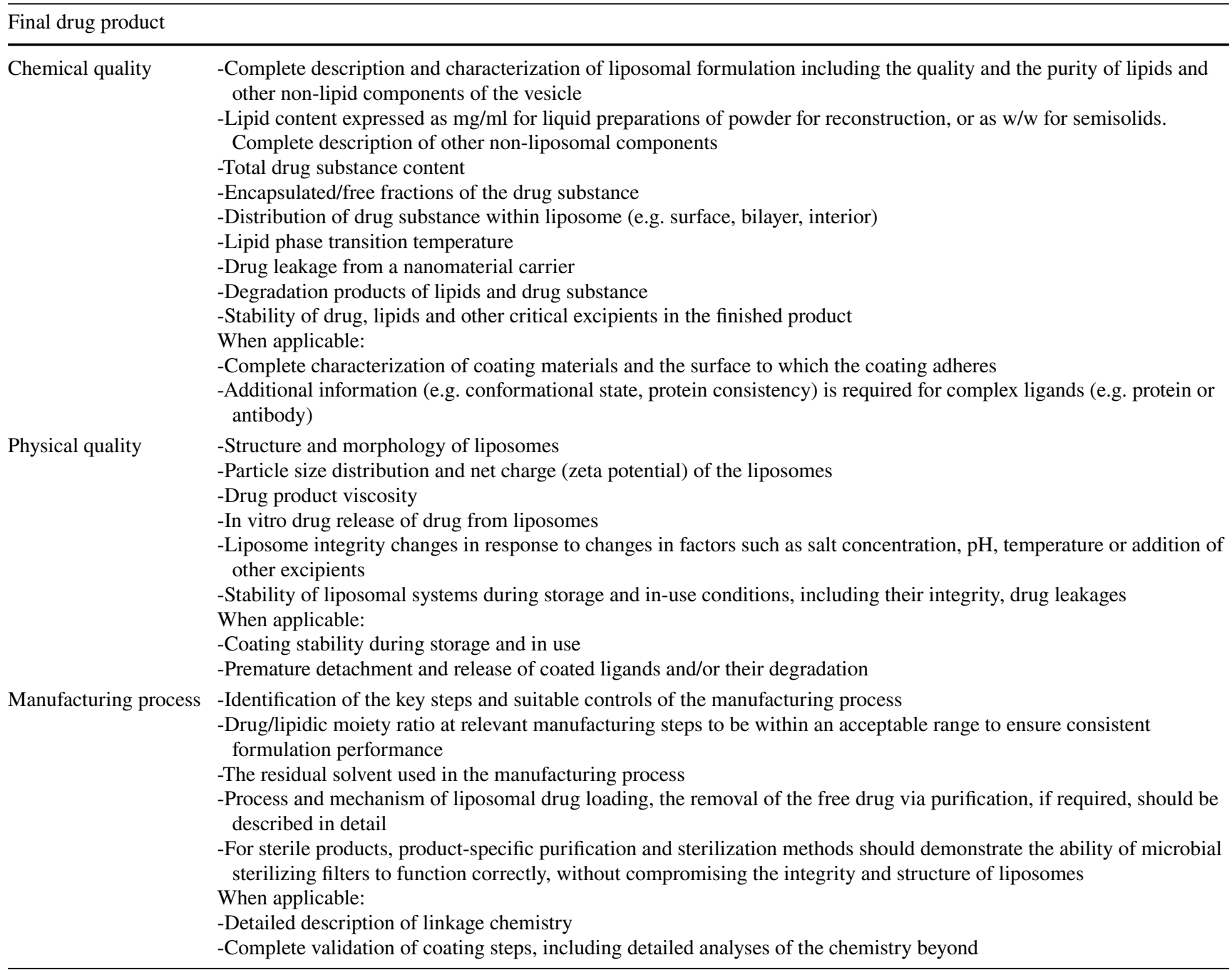


stability in both vehicles and physiological environments. The assessment of the quality of lipids used in the formulation has been particularly stressed since small changes in their spatial organization or purity have a huge impact on the technological and biopharmaceutical performances of the liposomal systems. When the liposomal surface is coated by a ligand, the nanosystem complexity is increased and, therefore, additional information should be provided (Table 1). In this light, a full characterization of coating materials and the surface to which the coating adheres should be performed to assess their impact on the nanosystems' performance [24]. The extent of the required studies varies based on the ligand type: the higher the ligand complexity (e.g. small molecules, peptides, proteins, antibodies), the larger the additional data the developers have to provide.

For the pharmaceutical development of new nanomedicine products, the research on in vitro biorelevant tests is still crucial due to the lack of sensitive analytical methodologies, validated experimental protocols and clear in vitro/in vivo correlations. Such criticisms are particularly relevant in the design of topical nanomedicine systems, considering also the additional information required by a regulatory agency to assess their benefit/risk balance. For example, the FDA requires developers to perform an in-depth investigation on the hair follicle penetration of the topical nanomedicine products, and on how the permeation profile of nanosystems and drugs is influenced by the skin conditions (e.g. intact, damaged, diseased) [23]. In this context, permeation kinetics studies (discussed in the last section of this work) can be useful to overcome the lack of knowledge on the skin permeation of nanosystems and to rationalize the design of both non-clinical and clinical studies. Indeed, they have been already accepted by regulatory agencies as a surrogate for testing the topical absorption of small molecules [27, 28]. For example, the EMA draft guideline on the quality and equivalence of topical products [27] opens the possibility, for complex formulations, to perform permeation kinetics (e.g. in vitro permeation studies) not only in the pharmaceutical development but also as supportive studies in the assessment of the therapeutic improvement/equivalence of the topical nanomedicine product with respect to conventional formulations applied on the skin. Similarly, such in vitro studies can provide helpful preliminary results to support the Comparison Clinical Pharmacology Studies required by the FDA when non-liposomal products are available on the market [28].

Based on such premises, the available information on the main techniques employed for the physicochemical characterization of vesicles, and the models to assess skin penetration are discussed in the following sections. The relevance of data provided by such methodologies for assessing the quality, efficacy and safety of the nanomedicine products is summarized in Table 2.

\section{Physicochemical characterization of liposomes}

Based on their intrinsic features of the formulation and the absorption route, the topically applied liposomal products are classifiable as non-biological complex drugs [20]. As other complex formulations, small changes in the critical quality attributes (CQA) have a substantial impact on the performance of in vivo and, therefore, an in-depth evaluation should be mandatory for driving the pharmaceutical development based on the target product profile (TPP) of the finished product [22]. Thus, as above mentioned, deep characterization of chemical features of excipients and their possible interactions with the drug is required for quality purposes in the definition of formulation space which can be rationalized with the help of Quality-by-Design (QbD) [29, 30].
Table 2 Application of physicochemical characterization methodologies and skin penetration models for assessing the quality, efficacy and safety of the nanomedicine products

\begin{tabular}{|c|c|c|c|c|c|}
\hline \multirow[t]{2}{*}{ Characterization } & \multicolumn{3}{|c|}{ Quality (Module 3) } & \multirow{2}{*}{$\begin{array}{l}\text { Preclinical } \\
\text { (Module 4) }\end{array}$} & \multirow{2}{*}{$\begin{array}{l}\text { Clinical } \\
\text { (Module } \\
5 \text { ) }\end{array}$} \\
\hline & $\begin{array}{l}\text { Pharmaceutical } \\
\text { development }\end{array}$ & $\begin{array}{l}\text { Quality } \\
\text { controls }\end{array}$ & $\begin{array}{l}\text { Post- } \\
\text { marketing } \\
\text { variations }\end{array}$ & & \\
\hline Dynamic light scattering (DLS) & $X$ & $\mathrm{X}$ & $X$ & & \\
\hline Deformability Index & $X$ & & $X$ & & \\
\hline Infrared spectroscopy & $X$ & & $X$ & & \\
\hline Differential scanning calorimetry (DSC) & $X$ & & & & \\
\hline In vivo models & & & & $X$ & \\
\hline Ex vivo models & $X$ & & $X$ & $X$ & $X^{(1)}$ \\
\hline Cell-based models & & & & $\mathrm{X}$ & \\
\hline In vitro skin permeation studies & $\mathrm{X}$ & & $\mathrm{X}$ & & $X^{(1)}$ \\
\hline In vitro membrane permeation studies & $X$ & $\mathrm{X}$ & $X$ & & \\
\hline
\end{tabular}

${ }^{(1)}$ Performed for supporting and/or rationalizing the design of clinical trials 
In the following sections, the most common physicochemical methodologies used for the characterization of topical liposomal formulations are discussed in the framework of this review. Of note, the techniques herein reported could be suitable also for the characterization of lipid nanoparticles (LNPs), the novel type of vesicles used to stabilize the mRNA in COVID-19 vaccines and that might be explored as a tool for nucleic acid delivery to the skin $[31,32]$.

\section{Dynamic light scattering (DLS)}

The mean size, size distribution and polydispersity of nanomaterials are QAs for their preclinical characterization [33] since they directly influence the biodistribution and, more specifically, skin interaction. To date, dynamic light scattering (DLS) is, from a regulatory point of view, the technique of choice for size determinations despite its low resolution, mainly because of its reasonable cost and ease of use [33, 34].

In DLS experiments, it is assumed that the nanomaterial is spherical. For the tests, a stable, monochromatic laser enters a cuvette made of disposable plastic or glass, containing a homogeneous and transparent suspension of the nanomaterial sample with no precipitate. Suspended nanomaterials move with Brownian motions and thus the laser light entering the cuvette undergoes a scattering that depends on the size of the suspended particles. The scattering, in turn, causes fluctuations of incident light fluctuations over time [35], which correlated to short decay intervals $(\tau)$ and used to determine the autocorrelation function which, for ideal monodisperse samples, is expressed by the following equation (Eq. 1) [36]:

$G(\tau)=1+b \cdot \exp \left(-2 D_{t} q^{2} \tau\right)$

In Eq. (1), $b$ is a characteristic constant of the specific DLS apparatus, $D_{t}$ is the translational diffusion coefficient and $q$ is a scattering vector accounting for the specific DLS system, expressed by (Eq. 2):

$q=\frac{4 \pi \cdot R I}{\lambda_{0} \cdot \sin _{2}^{\theta}}$

where $R I$ is the refractive index of the suspending medium, $\lambda_{0}$ is the laser wavelength in the vacuum and $q$ is the scattering angle. DLS instruments such as Malvern Zetasizer ${ }^{\circledR}$ plot the Raw Correlation Function (RCF) as a function of the delay time ( $\tau$; Eq. 3$)$ :

$R C F=G(\tau)-1$

The experimental fluctuations are autocorrelated and fit to an autocorrelation function using $D_{t}$ as an adjustable parameter. The obtained $D_{t}$ value is then used to calculate the hydrodynamic radius $\left(R_{H}\right)$ of the nanomaterial via the Stokes-Einstein equation (Eq. 4):

$D_{t}=\frac{k_{B} T}{6 \pi \eta \cdot R_{h}}$

Here, $k_{B}$ is Boltzmann's constant, $T$ the absolute temperature and $h$ the viscosity of the suspending medium.

The reported equations show that DLS outcomes are primarily determined by the viscosity of the suspending medium, by the features of the specific apparatus and by the refractive index of the sample [37-39].

It must also be specified that, in Eq. (4), $R_{H}$ is an equivalent size corresponding to the radius of the rigid sphere able to diffuse with the same speed of the sample [40]. However, nanomaterials in suspension are often solvated and, consequently, DLS can provide results that are only indicative of actual nanomaterial size.

All in all, the core components of DLS instruments are the laser, the sample and a light detector. Modern DLS instruments are oftentimes equipped with detectors placed at an angle of $173^{\circ}$ to catch possible backscattering, thereby helping detect the signals produced by the population of smaller particles [41].

In general, it can be asserted that DLS is fast and inexpensive, albeit with a low resolution, and is still considered a technique of choice in the pre-screening phase of nanomaterial production. Actually, the DLS technique used in "batch" mode has severe limitations. For instance, the intensity of the scattering depends on the sixth power of the radius. Consequently, a small number of large particles can mask larger populations of smaller particles, thereby resulting in misleading results for polydispersed samples since it is impossible to discriminate between populations of particles with similar diameters $[33,34]$ or to distinguish between small aggregates and larger particles [42].

Lately, new DLS instruments combine different and innovative technologies based on light scattering and allow full characterization of liposomes and colloidal dispersion systems. Specifically, Zeta sizer instruments, such as Nanosizer Ultra (Malvern Instrument, UK), measure particle size in a wide concentration range (up to $40 \% \mathrm{w} / \mathrm{v}$ ), with the possibility to select or create an ad hoc analysis method based on each sample. Indeed, by employing the built-in ZS explore software, the operator can select within a wide database of possible particles samples, each characterized by a different value of the refractive index (RI) and absorption (for liposomes, RI is 1.45 and absorption is 4.276). Likewise, it is also possible to select the values of the same parameters for the dispersant (e.g. in the case of water at $25^{\circ} \mathrm{C}$, RI is 1.33 and viscosity is 0.8872 poise at $25^{\circ} \mathrm{C}$ for water). With this instrument, a multi-angle dynamic light scattering 
analysis (MADLS) is also possible. Thus, in a MADLS single analysis, three different angles (back, side, forward) can be used to obtain a unique dimensional distribution curve (https://www.malvernpanalytical.com/en).

\section{Deformability index}

In the field of dermal/transdermal administration, the flexibility of liposomes is a crucial parameter to define the penetration properties [43, 44]. Liposome flexibility is associated with a high deformation ability, which is generally promoted if the mixture of lipids that constitutes the vesicles has a low phase transition temperature. The flexibility of liposomes is important because it is associated with the ability to promote skin accumulation and permeation of actives [45-47]. Indeed, it has been reported that highly flexible liposomes can squeeze through skin pores, and the amount of squeezing is correlated to the propension of vesicle bilayers to undergo deformation [48]. More in detail, the knowledge of liposome deformability can help to predict the ability of liposomes to permeate through/deposit into the layers of the skin $[19,49]$.

Liposome deformability can be quantified by the extrusion method, which consists of forcing the liposomes through a porous polycarbonate membrane of known pore size, in the order of tens or hundreds of nm, under a known pressure in the order of MPa [50,51].

The basic principle is that conventional liposomes, having a stiff bilayer, when forced through the membrane undergo fragmentation occluding the tiny pores of the membrane whereas flexible liposomes, being deformable, pass through the membrane maintaining their morphology [52]. In such a pressure-governed process, the flux (i.e. the amount of suspension extruded in a fixed time lap) is proportional to the relative vesicle/pore radii ratio and then, the deformation index (DI) can be calculated with the following equation (Eq. 5):

$D I=J \cdot \frac{d_{0}}{p} \cdot \frac{d_{0}}{\left|d_{1}-d_{0}\right|}$

where $J$ is the fraction of liposomes recovered after the extrusion process; $d_{0}$ and $d_{1}$ are the mean liposome diameters before and after extrusion, while $p$ is the pore size of the membrane.

Using similar experimental conditions, other authors proposed a simpler approach consisting of the expression of deformability as the reduction of vesicle diameters after extrusion [53]. Furthermore, the extrusion through a pore can be performed under a constant rate condition with the possibility to evaluate the force required to deform or break the liposomes [54].

Alternatively, to such extrusion-based methods, other approaches have been proposed based on the use of ESR
[55] or AFM techniques [56]. In particular, AFM measurement allows a real-time analysis of a curve force measured during the indentation of the probe on the liposome bilayer which can be used to determine Young's modulus of vesicles which is strictly related to the deformability of the nanostructure [56].

The DI has been correlated to the skin crossing ability of liposomes [57]. Moreover, for a fixed lipid composition, the inclusion of actives into the liposome bilayer can affect the deformability index and, consequently, the active molecule accumulation into the skin. Recently, Campani et al. showed that DPPC liposomes loaded with high amounts of CURC (15\% w/w) possess a high DI (77 vs 17-27 for lower CURC loadings). Consequently, the intercalation of CURC in liposome bilayer is associated with a less ordered structure and to vesicle ability to undergo deformation. This, in turn, results in the accumulation of CURC within deeper layers of the skin [57].

From a regulatory point of view, the DI may be considered a CQA of topically applied liposomal formulations. Due to the availability of reliable tests in the literature, its determination may be considered a useful strategy to waive the more expensive studies on animal models. Indeed, the DI determination can be used both to guide the pharmaceutical development in the selection of the best formulation and to assess batch-to-batch comparisons in the management of post-marketing variations. Even if the validation of this type of experiment may appear critical due to the complexity of the applied methodology [56], such tests are ideally feasible to be also eventually applied routinely in the quality control of topical liposomal systems.

\section{Infrared spectroscopy}

Infrared (IR) spectroscopy is a long-established analytical technique used for the characterization of a wide array of nanosystems and materials [58]. Infrared radiation refers to that part of the electromagnetic spectrum which lies between the visible and microwave regions and can be divided into three different regions: the near (NIR$13,000-4000 \mathrm{~cm}^{-1}$ ), the mid (MIR-4000-200 $\mathrm{cm}^{-1}$ ) and the far infrared (FIR-200-10 $\mathrm{cm}^{-1}$ ) named after their relation to the visible spectrum. From a general point of view, IR is vibrational spectroscopy that involves interactions between electromagnetic radiation and the material; this technique provides information about molecular structure and molecular interaction between different structures, through each functional group. Practically speaking, when an organic molecule is invested by an infrared frequency ranging between $500^{-1}$ and $4000 \mathrm{~cm}^{-1}$, the transferred energy is converted into vibrational energy [59]. A single molecule can vibrate by bending vibration, due to the 
variation of the bond angle with a common atom, or to a movement of a group of atoms with respect to the remaining portion of the molecule that remains fixed one with respect to the other. The deformation can also be symmetrical or asymmetrical and can occur along the plane on which the bond angle lies (scissoring or rocking motion) or outside that plane (wagging e twisting motion). On the other hand, a stretching vibration is due to the rhythmic stretching along the bond axis with a resulting modification of the interatomic distance. Thus, this vibration produces an oscillating electric field allowing the exchange of energy with electromagnetic waves. As described in more detail by Centrone [59], sample features, analyzed by IR, can be described in terms of a refractive index $(N)$ expressed by the following equation (Eq. 6):

$N(\lambda)=\mathrm{n}(\lambda)+i \cdots k(\lambda), 1$

where $\lambda$ is the light wavelength and $n$ and $\kappa$ are the parts of the index related to light scattering and absorption, respectively. The use of IR and Fourier transform infrared (FTIR) spectroscopy has attracted increasing interest to deeply characterize nanoscale systems of different nature, chemical composition and morphology [58]. FTIR provides a sort of "fingerprint" of the sample under examination and is able to show, for example, protein interaction with nanoparticle surface [60]. In the case of lipid nanovectors such as liposomes, FTIR can be used to grasp information about lipid composition, interactions between components (i.e. the presence of cholesterol in the bilayer) and their influence on drug encapsulation and stability [58]. Recently, FTIR spectroscopy analyses have been carried out to understand the influence of cholesterol in 1,2-dimyristoyl-sn-glycero3-phosphocholine (DMPC) and 1,2-distearoyl-sn-glycero3-phosphocholine (DSPC) liposome bilayers and/or the influence of liposome preparation methods, i.e. microfluidics and hydration methods on the molecular arrangement [61]. The analysis of IR spectra showed that the presence of cholesterol at the highest concentration leads to stronger interactions with lipid components (revealed by the presence of weaker peaks) especially in DMPC liposomes, while the preparation technique did not alter liposome characteristics [61]. FTIR spectroscopy was also used to investigate the interaction between skin components and liposome formulations designed for topical or transdermal delivery of actives compounds [62]. A research study showed that the topical application of transfersomes encapsulating meloxicam, a nonsteroidal anti-inflammatory drug (NSAID) used in pain management, altered the stratum corneum and increased its flexibility suggesting a more effective transdermal delivery compared to classic liposomes. In actual fact, the spectrum of the skin treated with the transfersome formulation was characterized in a shift of $\mathrm{C}-\mathrm{H}\left(\mathrm{CH}_{2}\right)$ asymmetric stretching peak to higher frequencies compared to the untreated skin [62]. Skin permeation studies confirmed that transfersomes provide a greater meloxicam skin permeation as a consequence of the disruption of the outer layer of the skin [62].

In addition to FTIR technique, attenuated total reflection (ATR)-FTIR spectroscopy is also used for liposomes characterization. This technique allows a higher penetrating depth of the waves in the sample (up to $5 \mathrm{~mm}$ ) and reduces the issues related to sample preparation and reproducibility compared to IR [63]. In a recent study, Campani et al. have developed and characterized different lipid nanocarriers to enhance the accumulation of vitamin K1 on the skin; thus, the authors investigated the possible effects of these formulations on the arrangement of the major constituents of the stratum corneum (i.e. the intercellular lipids and keratins), after topical application [19]. The analysis of the ATRFTIR spectrum, of $\mathrm{CH}_{\mathrm{x}}\left(2988-2828 \mathrm{~cm}^{-1}\right)$ stretching and scissoring (1480-1440 $\mathrm{cm}^{-1}$ ) regions, showed the lack of significant modification in the lipid organization suggesting that there was not an alteration of the orthorhombic conformation of lipids after the administration of lipid vesicles [19]. Moreover, the determination of a significant decrease of the ratio between the maximum intensity of deconvolution of $v_{\text {asym }} \mathrm{CH}_{2}$ and $\mathrm{v}_{\text {sym }} \mathrm{CH}_{2}\left(\mathrm{H}_{2920 / 2850}\right)$ values, related to the amount of lipid in the stratum corneum, suggested an enrichment of the lipidic components of the stratum corneum and the formation of a more ordered structure [19]. These results corroborated the hypothesis of the penetration of intact vesicles within the stratum corneum, which enrich the lipidic skin components and increase the order of the structure. Finally, the deconvolution of amide I showed the presence of nine hidden peaks characteristics of keratin conformations and ceramides, as well as the bands assigned to $\alpha$-helices and $\beta$-turns structures $\left(1660-1650 \mathrm{~cm}^{-1}\right.$ and $1668-1682 \mathrm{~cm}^{-1}$, respectively), confirming that the exposure of skin samples to all the tested formulations did not alter the distribution of the secondary structures of stratum corneum proteins [19]. Thus, deep characterization of the formulations can help the assessment of the quality of the designed systems in the pharmaceutical development step. Moreover, providing deep knowledge of the possible interactions occurring among formulation components and stratum corneum, the FTIR data can result helpful in the management of post-marketing variations.

\section{Differential scanning calorimetry (DSC)}

Differential scanning calorimetry (DSC) is a thermoanalytical technique used to detect the thermal events of a wide array of materials, such as polymers, drugs and biological samples. DSC outcomes are useful to complement infrared and Raman spectroscopic data. DSC experiments are 
performed by imposing a pre-programmed temperature program on the samples, for example by utilizing isothermal or dynamic conditions. Two capsules, generally made of aluminium, are placed in a DSC apparatus; one contains the sample under examination and another, often empty, is used as a reference. During the test, the difference between the heat flows emitted/absorbed by the sample and the reference capsule undergoing the same thermal history is measured. The obtained differential heat flow allows to calculate (at constant pressure) the heat capacity of a material, expressed as cal/C or $\mathrm{J} / \mathrm{C}$. This amount, normalized for the mass of the specimen, allows to obtain the specific heat capacity $C_{p}$ at constant pressure, which is expressed as cal/(g C) or cal/(mol C), as shown hereafter:

$C_{p}=\left.\frac{1}{m} \cdot \frac{\partial q}{\partial T}\right|_{p=\operatorname{cost}}$

where $m$ is the mass of the sample, $q$ the heat evolved during the test, $T$ the temperature and $p$ the pressure. Correspondingly, the specific heat of a given thermal transition, expressed as $\mathrm{J} / \mathrm{g}$, is given by:

$\Delta H=\int_{T_{1}}^{T_{2}} C_{p} d T$

Unless otherwise needed, DSC tests are carried out under an inert atmosphere to avoid possible unwanted reactions with oxygen, for instance.

DSC is also used to study the thermal transitions of phospholipids, as such or arranged as bilayers and liposomes [64]. Bilayers exhibit a broad polymorphism, which is mainly related to the gel-to-liquid crystalline phase transition, and this thermotropic behaviour is associated with an endothermic event [65]. Actually, the study of thermal transitions of phospholipid bilayers by the changes of enthalpy $(\Delta \mathrm{Hcal})$ during the transition process is of great importance not only to characterize the thermotropic predilection of liposomes but also to rationally design lipid drug carriers based on their thermal behaviour [65].

After a temperature increase, liposome bilayers transition from the ordered gel state to the disordered fluid lamella. This is associated with a promoted mobility between the hydrocarbon chains of phospholipids due to a decrease in the inter-chain van der Waals interactions. This, in turn, relates to a general decrease in the stability of the system [66]. The gelto-liquid crystalline transition of pure phospholipids appears as a sharp endothermic peak, due to favourable van der Waals interactions of lipid acyl chain resulting in high cooperativeness of the molecules [65]. The enthalpy associated with this transition, expressed as $\mathrm{J} / \mathrm{mol}$ or $\mathrm{J} / \mathrm{g}$ of phospholipid, is quickly obtained from peak area in a DSC thermogram.
Among the phospholipids used to produce liposomes, dipalmitoylphosphatidylcholine (DPPC) is one of the most often characterized by DSC due to the sharp, well-defined endothermic peak associated with the phase transition around $40{ }^{\circ} \mathrm{C}$.

The understanding of the interactions occurring between active pharmaceutical ingredient (API) and liposome membrane plays a paramount biological role since APIs can reach the target site after membrane crossing. In a recent study [67], the interplay between levothyroxine (3,5,3', ' $^{\prime}$-tetraiodothyronine; L-T4), a L synthetic thyroid hormone, and DPPC has been studied by FTIR and DSC. The outcomes of the thermo-analytical experiments shed light on how L-T4 structurally affects DPPC membranes. Indeed, DSC results indicated that increasing amounts of L-T4 caused a decrease in temperature and enthalpy of transition along with an apparent endothermic peak broadening. This hints at a drop of the cooperativity among lipid segments in DPPC due to L-T4 intercalation within DPPC lipid domains [68-71], therefore disturbing the hydrophobic interactions of lipids [72]. This in turn suggests a probable change of DPPC bilayer packing because of an increased membrane disorder induced by L-T4 intercalation, probably in interfacial and hydrophobic domains of the bilayer [73]. Similar results were also obtained in a recent paper [66] where DSC outcomes indicated that tocopherol loading in DPPC liposomes was associated with a loss of membrane order and an increase in membrane deformability.

In this same direction, Campani et al. have recently produced DPPC liposomes loaded with different amounts of curcumin (CURC) [57]. This molecule, which is strongly lipophilic, significantly interferes with the packing of the acyl chains of the DPPC. This leads to an increase in the fluidity and deformability index of the liposomes, and in turn this leads to a promoted deposition in the epidermis [57]. It must be emphasized that, in this work, thermal transitions in liposomes were detected directly in aqueous suspension, using the same volume of pure water as a reference. This strategy is useful since it allows to find out thermal features of liposomes in the conditions of use. On the other hand, the thermal properties of only a handful of phospholipids can be detected, since the temperature range in this case is necessarily limited (approximately from 10 to $80^{\circ} \mathrm{C}$ ). From a regulatory point of view, the relevance of data generated on a liposomal system that differs in composition with respect to the formulation under development/marketed may present some concerns. However, DSC data can provide information essential to drive some formulation choices and, therefore, they should be considered important supportive data in pharmaceutical development. 


\section{Assessment of percutaneous permeation and biological effect}

Depending on the pathology to be treated, topically applied active agents might be intended for local action within the skin or systemic absorption. The target site might also be an anatomical district below the skin (e.g., muscles or joints). In such a case, the drug would need to cross all the skin layers, being available for systemic absorption, and ideally showing higher retention in the target area due to the proximity of the administration site. In all these cases, assessing the skin permeation of drug molecules (and nanocarriers) is a crucial step in the development of an effective formulation.

As human studies would only be performed in the final steps of development of a new topical (nano)formulation, several in vitro, ex vivo and in vivo models have been proposed to analyze the percutaneous absorption of drugs. A fundamental question stands before the vast number of skin models developed by companies and researchers: what is the correlation with human studies? Answering this question would pave the way to standardized test protocols accepted by regulatory authorities, thus reducing the time and cost of the development of new topical nanomedicines.

In the next sections, we will provide an overview of the skin models employed for the assessment of nanocarrier permeation in the skin, organizing them as in vivo, in vitro, cell-based and synthetic membranes.

These assays should be designed and combined not only for defining the quality attributes of the drug products but also to verify the fate of the formulation upon topical application. The biodistribution of the active agent, as well as of the other components, should be elucidated, in analogy with current regulatory guidelines related to parenteral nanomedicines. Currently, most of the research efforts are directed towards the determination of the active molecule(s) within and beneath the skin, while little emphasis is put on the local/systemic quantification of the other formulation components. As an example, the feasibility to quantify cationic lipids such as DOTAP was reported [56]. However, in most of formulations reported in the literature, the phospholipids used in topical liposomes are similar or identical to the skin lipids. This poses a technical limitation to the analysis of their permeation, for which either the use of labelled lipids or advanced techniques such as secondary ions mass spectrometry (SIMS) bioimaging is required [74, 75]. The simultaneous tracking of the active ingredient and the other components would allow gaining information on the mechanisms of dermal permeation. Moreover, including such analysis in the development process would better demonstrate the safety of the designed nanosystem.

\section{In vivo models}

Despite the significant advancements of in vitro models in the framework of 3R research (Replacement, Reduction and Refinement), the tests performed on laboratory animals are still mandatory to assess the pharmacokinetics, pharmacodynamics and toxicology of novel pharmaceutical products (including nanomedicines) before proceeding with clinical studies [76]. In the case of topically applied products, the differences between human and animal skin should be taken into consideration when analyzing the results of a skin permeation study in vivo. A wide number of species have been used to test drug permeability in vivo, including mice, rats, guinea pigs and pigs [77]. However, the lipid composition (free fatty acids, ceramides, triglycerides) and organization, as well as the thickness of the stratum corneum and epidermis, vary greatly between species, causing differences in the barrier properties and sub-optimal correlation between animal and human studies [78]. In addition to the speciesspecific skin properties, inter and intra-individual variability arising from differences in age, sex and anatomical site should be considered when planning in vivo (and in vitro) experiments [79].

Among the laboratory animals, pigs have a skin structure and barrier properties that best reproduce the human skin [80, 81]. However, due to maintenance costs and ethical concerns, the use of pigs and minipigs is more frequent in vitro than in vivo [82]. Rodents, which are the most employed mammals in biomedical research, are widely used also in the field of topical pharmaceutical products testing, for their ease of handling and relatively low cost. However, the skin of mice and rats is characterized by a significantly higher density of hair follicles compared to human skin, so hairless strains are more relevant [83]. On the other hand, studies on normally haired rodents can provide useful information on the penetration pattern through hair follicles, which is required not only for developing medicinal products but also to demonstrate the safety of other healthcare products, even if the amount of permeated drug is overestimated [84, 85]. Thus, the biological effect of a topically applied drug in rodents might not be replicated in humans due to the lower permeated dose. However, preclinical data can provide information on the biodistribution of the absorbed free/loaded drug fractions [21].

In the case of topically applied liposomal formulations, the use of ex vivo models employing viable human skin is being developed to fill the gap between in vivo results obtained by animal models and possible behaviour after administration in man. To maintain the viability of the excised tissue, human skin might be perfused and oxygenated using a peristaltic pump and tubing connected to a cannulated artery [86]. Despite the higher complexity of 
the system, defined as isolated perfused human skin flap (IPHSF), the last decade witnessed an increase in its use, also for testing lipid-based nanosystems [87]. The knowledge on the fate of the drug carried by nanocarrier in these models can help the design of the clinical trials mitigating the risk of their failure either in terms of efficacy or safety.

\section{In vitro skin permeation studies}

Biopharmaceutical performances and efficiency of both topical and (trans)dermal drug delivery systems are generally demonstrated by in vitro skin penetration studies, usually performed using a diffusion cell with donor and receiver compartments separated by a membrane.

According to EU Guidelines on quality of topically applied formulations $[27,88]$, the in vitro skin permeation experiments, which permit a quantitative determination of the amount of the penetrated drug after cutaneous administration, are not only useful in the definition of the formulation space and related CQA but also should support the design of clinical trials in which the output is mainly based on clinical evaluations. As an example, the in vitro experiments can provide an estimation of the variability of the permeation pattern of a drug [89], which can be modified by a liposomal formulation and therefore it will result useful for definition of the potency (number of volunteers to be recruited) of the clinical trial.

The human skin is clearly the most relevant membrane model, and in vitro permeation studies across human epidermis or dermatomed human skin-either excised from surgical reduction or from a cadaver-are considered the gold standard to evaluate a topical drug delivery system [90]. However, the use of human skin is affected not only by ethical and regulatory obstacles but also by limitations related to the physiological variances between samples from both different donors and different anatomical districts of the same subject [91]. For these reasons, researchers have investigated the use of skin from other animals, and a large amount of experimental data was generated in the attempt of predicting human percutaneous absorption.

Currently, the in vitro topical delivery through lipid vesicles should be studied on human skin or pig skin, as prescribed by the FDA and EMA guidelines for other consolidated formulations.

Besides the different skin sources, the choice of an appropriate in vitro model can be affected by several factors which include the preparation technique and its storage conditions as well as the experimental setup. In this context, the most common skin preparation methods include full-thickness skin or split-thickness skin (dermatomed to about $0.5 \mathrm{~mm}$ ) as specified by the Organization for Economic Cooperation and Development (OECD) guidelines. In addition, heatseparated epidermis and trypsin-isolated stratum corneum have been largely used in percutaneous absorption studies $[92,93]$. It should be also mentioned that human and pig skin can often be used after frozen storage, differently from rodent skin that is usually used fresh [94]. In the case of the use of frozen skin, the acceptable storage period has to be established using appropriate methods which allow the integrity and maintenance of the barrier's properties of the membrane. The control is usually based on the measurement of physical properties such as electric resistance or trans-epidermal water loss (TEWL), eventually coupled with histological analysis [95].

Regarding the experimental design, most of the transdermal penetration and permeation studies are carried out by using a vertical Franz diffusion cell apparatus, which is reproducible and remarkably simple to operate [96]. It consists of a top chamber (donor compartment) where the tested formulation is applied, and a bottom chamber (receptor compartment) filled with an appropriate fluid, that should be selected to mimic the physiological environment and to provide adequate drug solubility. The skin membrane is placed between the two compartments, so that the stratum corneum is facing the donor compartment. The drug permeation rate through the skin is obtained by measuring the amount of drug recovered into the receptor compartment over time by means of an appropriate analytical method. Conversely, the drug penetration into the skin layers is detected by extraction after stratum corneum tape stripping and, possibly, epidermis and dermis separation.

In the field of liposomal dermal and transdermal drug delivery, the skin from human or animal sources has been also largely used in vitro with diffusion cells [97]. Various vesicular formulations have been tested on human skin as carriers for different drugs, mostly using Franz diffusion cells and/or tape stripping procedures [98-101].

As discussed in the previous section, a wide range of animal species is used for permeation studies, with pig being one of the most attractive due to the skin physiological similarities with humans [94]. As a cheap and readily available source of skin specimens, pig skin from slaughterhouse waste has been selected by several research groups as an alternative model to human skin for testing liposomal formulation. Importantly, using such a supply channel has an added ethical value. These models are especially useful for tape stripping experiments, where the drug penetration into the uppermost skin layers is investigated [102]. The skin from pig ear could be a suitable model to assess the distribution of liposome-loaded drugs within the skin or across the skin. As an example, Rukavina et al. demonstrated that liposomal encapsulation of azithromycin allowed its accumulation in the skin, limiting the transdermal penetration and potentially reducing the systemic exposure [103]. In the last decades, deformable vesicles, such as transfersomes and ethosomes, have been reported to improve 
the penetration of several drugs through the skin of pig and rabbit ears [49, 104-106]. Indeed, pig stratum corneum is the most similar to human stratum corneum in terms of lipid composition, but it presents a marked difference in terms of thickness. Since the stratum corneum is considerably thinner in younger pigs than adult pigs, newborn pig skin has been tested as a more human-relevant alternative than adult pig skin [81]. In 2005, the use of newborn pig skin was also pioneered for testing the percutaneous absorption of liposomes loaded with tretinoin [107]. Since then, several investigations on classical liposomes and innovative vesicles have been carried out with skin excised from newborn pigs which died for natural causes [108-111]. Besides the consolidated use of human and pig skins, also the pinna rabbit skin could be considered a valid alternative. Indeed, it shares with the pig skin the lack of ethical issues since it can be retrieved in a slaughterhouse and has been used for the characterization of several nanocarriers including lipid vesicles $[49,106$, 112-116]. Furthermore, the rabbit is widely used as in vivo model to assess the safety of topical formulations (skin irritation test, EN ISO 10993-10:2002).

\section{Cell-based models}

Cell-based skin models are stratified cell cultures composed of one or more skin cell types, with or without an extracellular support material [117]. Skin cells are cultured in vitro in specific conditions to promote growth in a way that mimics the histological organization and the barrier function of the skin [118]. Originally, the production of skin substitutes from cultured cells was investigated in the context of tissue engineering, with the main aim of providing artificial tissue for transplantation to patients having large areas of the skin damaged by burns or injuries. More recently, the use of cell-based skin models (also referred to as human skin equivalents, HSE) for drug and chemical testing has gained importance, mostly due to the need of reducing the use of animal models in biomedical research. Due to the presence of live and metabolically active cells, these models are ideal to assess skin irritation, corrosion, phototoxicity and-with some limitations that will be discussed - transdermal permeability. Importantly, reconstructed human epidermis (RHE) systems have been validated and included in the OECD guidelines for testing skin irritation (test no 439), corrosion (test no 431) and phototoxicity (test no 438) (https:// www.oecd.org/). In this light, cell-based skin models find application as supportive studies in the preclinical part of the authorization dossier for the toxicological assessment of both formulation and its components. Indeed, especially when exogenous lipids are included in the liposomal formulation, the use of cell-based skin models can be useful to enlarge the available information on their toxicological pattern to provide a better interpretation of the results of preclinical studies.
The main cell types required to reproduce the two viable layers of the skin, epidermis and dermis, are keratinocytes and fibroblasts, respectively. While fibroblasts are easier to cultivate and expand in vitro, keratinocytes are characterized by a slower proliferation rate and require particular conditions. One of the first methods developed to promote keratinocytes growth consisted in seeding them on a feeder layer of gamma ray-irradiated mouse fibroblasts [119]. Such treatment blocks the proliferation of fibroblasts, which would still be able to provide keratinocytes with growth factors and signals to stimulate proliferation. After this first report in 1975, almost 50 years of research led to the modern methods of artificial skin engineering that use human cells, avoid components of animal origin (such as bovine serum), employ an optimal balance of salts and growth factors, can include other cell types (such as melanocytes and immune cells), can be supported by chemical substrates (such as fibronectin and collagen) alternatively to the fibroblast layer and are cultured at the air-liquid interface to promote the differentiation of keratinocytes and formation of defined epidermal sublayers [120].

The number and complexity of HSE that are commercially available or built in-house by research groups are growing. Their development is usually paired with the analysis of histology, lipid composition and/or the assessment of the barrier function by tracking the permeation of model compounds, or by trans-epithelial electrical resistance (TEER) and/or TEWL measurements [118]. In most cases, the histological analysis of commercially available HSE reveals an epidermis with clearly defined sublayers (stratum basale, stratum spinosum, stratum granulosum and stratum corneum). Conversely, the lipid composition is altered in HSE compared to human skin, with increased fractions of unsaturated free fatty acids and ceramides, altered ceramides composition and uneven distribution of lipid species [121, 122]. Such differences in lipid composition, together with the absence of appendages and other structures, are thought to be responsible for the impaired barrier function compared to excised human or animal skin. Indeed, several comparative studies have shown a generally higher permeation of drug molecules across in vitro cell-based models as compared to in vitro skin models $[123,124]$.

To date, the use of cell-based in vitro models to test the skin permeation of topical liposomes has been limited. In one of the earliest reports, Kuntsche and colleagues observed a higher permeability of different lipid nanoparticles across a rat epidermal keratinocytes organotypic culture, compared to the excised human epidermis [125]. In another work, the skin permeation of acyclovir and chloramphenicol liposomes and solutions were assessed using a commercial RHE or a synthetic membrane [126]. Of note, the RHE experiment only showed minimal differences in permeation when the drugs were administered as solutions vs liposomes, while 
the synthetic membrane was able to detect a significant difference between the formulations. Cell-based in vitro models have also been used to assess the permeation of nanoparticles of different nature. For instance, a detailed description of the method for the analysis of gold nanoparticles permeation through a full-thickness human cell-based skin model was published [127]. More recently, Pinto et al. employed an RHE model to assess the permeation of nanostructured lipid carriers (NLC) loaded with retinyl palmitate and tocopherol, two ingredients commonly used in cosmetic products [128]. While not providing a direct comparison, the authors state that the RHE might have a weak barrier function, as the permeation of tocopherol was significantly higher than in previous studies, where native human skin was employed.

Overall, the use of HSE for the evaluation of nanocarrier permeation is not yet fully validated, and the use of skin in vitro and synthetic membranes remains prevalent. Some experts also suggest that the use of HSE for drug permeation studies cannot be recommended [118]. However, the implementation of HSE could provide precious information regarding the interaction of liposomes with live cells organized in a tissue. In these systems, the role of specific transporters or membrane proteins in nanoparticles transport could be analyzed by silencing or overexpressing such genes. Moreover, HSE models - being composed of live cells-are ideal to evaluate the toxicological profile of liposomes and their cargo in terms of phototoxicity, irritation and corrosion (according to the OECD guidelines cited previously) as well as their pharmacological activity on skin cells. For instance, the photoprotection of a liposome-based product was assessed on RHE by analyzing the sunburn cells formation and the expression of proinflammatory genes upon UV irradiation [129]. Similarly, an HSE was employed to demonstrate that liposomes loaded with antioxidants could reduce the cell damage triggered by a mustard gas analogue. When developing a topical 5-fluorouracil liposomal formulation for the treatment of basal cell carcinoma, Cadinoiu et al. determined its irritation potential on normal cells using a commercial RHE [130]. Expanding the discussion to nanoparticles of different nature: the silencing efficacy of siRNAs conjugated to gold nanoparticles was assessed (together with their permeation) using a commercial RHE [131]. A reconstructed skin model composed of human keratinocytes supported by a fibroblasts layer embedded in a collagen matrix was used to assess the phototoxicity of quercetin loaded NLC [132]. The genetic toxicity of silica nanoparticles could be determined on RHE by adapting the micronucleus assay [133]. Moreover, RHE could be used to determine the effect of a nanoparticle treatment on the skin barrier function, measuring the distribution of an impermeable dye before and after the application [134].

In conclusion, despite the great advances in the last decades, it is our opinion that in vitro cell-based models are not yet mature to be used as the sole tool to test the skin penetration of liposomes and, more in general, of nanoparticles. As discussed in the previous sections, their use could provide important information on the mechanism of diffusion across strata of live cells, but such data should be complemented by the results from transdermal penetration studies using skin explants of humans or pig origin. On the other hand, HSE are an exceptional instrument to assess the pharmacological and toxicological effect of novel topical nanomedicines, providing more significant information compared to $2 \mathrm{D}$ cell cultures.

\section{In vitro membrane permeation studies}

Ex vivo and in vitro skin permeation models have some disadvantages, such as the low storage stability of skin specimens and high intra- and inter-individual skin variations (different thickness and hair density). For these reasons, over the last three decades, several attempts have been made to develop in vitro models based on artificial mimetic membranes that could represent a reproducible alternative to study the physicochemical mechanisms of drug permeation through the skin. Also, permeation studies across synthetic membranes could support the design of the liposomal formulation, especially if a relationship between the permeation flux through the artificial membrane and in vivo/ ex vivo/in vitro skin penetration is demonstrated. Furthermore, although the use of synthetic membranes is not sufficient for estimating the biopharmaceutical performances of a topical formulation, they can be usefully applied for quality purposes. Indeed, they can be used to assess the drug release as recently established by EU Guideline on quality of semisolid preparations [27] and issued in USP (monograph $<1724>$ Semisolid drug products - performance tests). They are also particularly relevant for comparing the bath-to-batch equivalence and/or for managing postmarketing variations.

The most studied artificial membrane can be classified in non-lipid-based models and lipid-based models.

\section{Non-lipid-based models}

Single-layer silicone membrane and microporous membranes, based on pure cellulose acetate, cellulose and polysulfone, have been tested as suitable substitutes to determine the permeability of drugs through different biological membranes [135-137].

However, while these artificial membranes could be used to predict the permeability of lipophilic compounds through biological barriers, they demonstrated a very low correlation when hydrophilic compounds are tested [138].

For this reason, more sophisticated artificial membranes have been developed. Strat-M® is a multi-layer synthetic 
membrane engineered to mimic human skin. It is composed of a thin top layer containing a proprietary blend of synthetic lipids (e.g. ceramides, cholesterol, fatty acids) that resemble the lipid composition of the human stratum corneum. Two lower polyether sulfone porous layers on top of a polyolefin non-woven layer create a morphology similar to the epidermis and dermis. In the last 5 years, an increasing number of studies recommended this artificial membrane in the evaluation of topical nanoformulations.

Touti et al. used Strat-M® and Raman microscopy for assessing skin penetration of multi-lamellar liposomes prepared from soybean lipids and Tween 80 (and eventually DOTAP or DPPG) [139]. They found that Strat-M® penetration is a complex mechanism depending on the mean diameter, zeta potential and flexibility of multi-lamellar liposomes. Most importantly, a correlation between Strat$\mathrm{M} \circledast$ and dog excised skin was established.

Strat-M® was also used to estimate the transdermal delivery of tocotrienol loaded in ethosomes [140]. The cumulative amount of tocotrienol permeated across this artificial membrane $\left(132.38 \pm 10.84 \mu \mathrm{g} \mathrm{cm}^{-2}\right)$ was significantly higher than those obtained using heat-separated human epidermis $\left(1.73 \pm 0.29 \mu \mathrm{g} \mathrm{cm}^{-2}\right)$ or full-thickness human skin $\left(1.03 \pm 0.24 \mu \mathrm{g} \mathrm{cm}^{-2}\right)$. This difference is ascribed to the presence of overlapping and tightly bound corneocytes in the skin, a feature that is not mimicked by the synthetic membrane. Interestingly, also the difference observed between the two biological membranes is significant, suggesting that the hydrophilic dermal layer represents a barrier for the diffusion of ethosomes.

Recently, cationic liposomes have been prepared using pyrrolidinium surfactants containing a hydroxyethyl fragment in the head group [141]. Transdermal delivery of liposomal ketoprofen has been tested in vitro using Strat-M ${ }^{\circledR}$ and Wistar rat skin, respectively. In both experiments, modified liposomes improved ketoprofen transdermal diffusion compared to conventional phosphatidylcholine liposomes. However, a higher amount of drug diffused through the rat skin, compared to the Strat-M® membranes. Therefore, while both systems could be employed to compare the permeation of different formulations, absolute values of permeated drug and flux might vary significantly.

\section{Lipid-based model membranes}

The Parallel Artificial Membrane Permeability Assay (PAMPA) was initially designed to predict the passive gastrointestinal absorption of drugs [142], and it was then modified to assessing drug permeability across other biological barriers, including the skin [143].

In original PAMPAs, a hydrophobic filter coated with a lipid mixture (phosphatidylcholines) separates a donor compartment containing the drug formulation and an acceptor filled with buffer solution. This resultant PAMPA sandwich is incubated (with or without stirring) at a controlled temperature for a given time to allow the permeation, and the drug amount in the acceptor is determined by a microplate reader. Skin-PAMPA models were developed by adding synthetic ceramides, cholesterol and stearic acid in the filter coating, as chemical compounds simulating the stratum corneum [144].

Casamonti et al. used Skin-PAMPA to evaluate the skin permeability of resveratrol-loaded liposomes and nanostructured lipid carriers. Skin-PAMPA revealed an increase in the skin permeability of resveratrol when loaded into liposomes [145].

The same artificial model membrane was employed to evaluate the permeability of berberine chloride loaded into escin-based liposomes. However, permeation data obtained using rabbit ear skin indicated a poor correlation with that obtained using Skin-PAMPA [114].

For their artificial nature, the synthetic membranes here described are highly homogenous in terms of thickness and composition. This provides higher reproducibility and consistency of permeation studies results compared to in vitro skin experiments. Moreover, numerous products are available in convenient 96-well formats suitable for high-throughput screening of many nanocarriers, representing an ideal tool for the initial formulation stages. On the other hand, synthetic membranes have seldom demonstrated a strong correlation with in vitro or in vivo permeability data, being more suitable for comparative studies between formulations. In addition to the structural differences with biological skin, the lack of some processes like metabolism, distribution and excretion further reduces the correlation with the in vivo conditions.

In conclusion, synthetic membranes are ideal for highthroughput screening of new nanoformulations in the initial phase of development. Cell-based in vitro models could provide useful information on the interaction of liposomes (and nanocarriers in general) with live cells organized in a 3D tissue. In vitro skin models are undoubtedly the most employed system to assess the permeation of liposome-loaded drugs. For this reason and an easier access to the technology, in vitro skin permeation experiments are increasingly taken into consideration by regulatory agencies for the evaluation of new pharmaceutical products. In vivo experiments in rats allow to study of some features that are only present in a living being, such as the drug clearance by dermal microcirculation, but data should be critically analyzed in light of the important differences between human and animal skin. Lastly, the pathological alterations of the skin barrier should be considered when testing a (nano)formulation intended to be applied on damaged areas, and appropriate in vitro or in vivo models of the disease should be employed. 


\section{Conclusion}

Despite the literature supports the application of topically applied liposomal systems - and more broadly of nanosystems-to improve the therapeutic performance of some active ingredients after topical applications, few liposomal-based products (e.g. a conventional liposome intended for the localization in the stratum corneum of econazole) reached the European market. Due to the higher developing/manufacturing costs, the rationale of a nanomedicine product strongly depends on the demonstration of its clinical superiority versus the therapeutic alternatives available on the market. In our opinion, a topical nanomedicine product should be superior in efficacy to other topical products and/ or in safety than systemic medicinal products authorized for the same therapeutic indication. In this light, topical nanomedicine products should follow full or hybrid applications to obtain marketing authorization. The extent of preclinical and clinical data and, consequently, the pre-authorization investment that the applicant has to provide are linked to the availability of reliable methods to assess and characterize the liposomal systems in vitro. The lack of robust in vitro studies to be used in place of clinical studies can be one of the causes of the difficulties for topical nanomedicines in reaching the market. Both in the case of first-in-man products and copies, the lack of well-established in vitro/in vivo correlations for topically applied nanosystems, together with the challenge of characterizing them, affects the assessment of their technological and biopharmaceutical properties.

It is noteworthy that the development and application of physicochemical characterization methods and skin permeation models can be very useful during the whole life cycle of a liposomal product. First, in the pre-marketing phases, for the assessment of drug product quality profile and the design of clinical studies. Secondly, once the product is authorized, to evaluate the impact of post-marketing variations and to support the pharmaceutical quality control system. Currently, the benefit/risk assessment of the product remains linked to case-by-case criteria that limit information sharing, the determination of the significance of the "intent to treat" for new medicines and the comparison among products expected to be therapeutic alternatives. In this context, a first step towards the harmonization of quality standards may be the application of regulatory provisions released for parenteral liposomal systems also to topically applied ones.

In conclusion, the non-consolidated regulatory framework on topically applied nanomedicine is strictly related to the poor number of requests for marketing authorization and vice versa. Thus, further studies dedicated to both penetration mechanisms and definition of the quality product profile are required to favour the development of such products.
Author contribution Work conception and design: FC, CS, GDR; writing - original draft preparation (introduction): FC, UMM, MS; writing - original draft preparation (regulatory part): FC, UMM; writing-original draft preparation (physicochemical characterization part): VC, MB, SF, GDR; original draft preparation (percutaneous permeation and biological effect part): MS, FL, CS; all authors reviewed the results and approved the final version of the manuscript.

Data Availability Not applicable.

\section{Declarations}

Ethics approval and consent to participate Not applicable.

Consent for publication All authors have read and agreed to the published version of the manuscript.

Conflict of interests The authors declare no competing interests.

\section{References}

1. Kováčik A, Kopečná M, Vávrová K. Permeation enhancers in transdermal drug delivery: benefits and limitations. Expert Opin Drug Deliv. 2020;17:145-55.

2. Roberts MS, Mohammed Y, Pastore MN, Namjoshi S, Yousef S, Alinaghi A, et al. Topical and cutaneous delivery using nanosystems. J Control Release. 2017;247:86-105.

3. Pireddu R, Schlich M, Marceddu S, Valenti D, Pini E, Fadda AM, et al. Nanosuspensions and microneedles roller as a combined approach to enhance diclofenac topical bioavailability. Pharmaceutics. 2020;12:1140.

4. Schlich M, Lai F, Murgia S, Valenti D, Fadda AM, Sinico C. Needle-free jet injection of intact phospholipid vesicles across the skin: a feasibility study. Biomed Microdevices. 2016;18:67.

5. Monteiro-Riviere NA, Inman AO, Zhang LW. Limitations and relative utility of screening assays to assess engineered nanoparticle toxicity in a human cell line. Toxicol Appl Pharmacol. 2009;234:222-35.

6. Zhang LW, Yu WW, Colvin VL, Monteiro-Riviere NA. Biological interactions of quantum dot nanoparticles in skin and in human epidermal keratinocytes. Toxicol Appl Pharmacol. 2008;228:200-11.

7. Ryman-Rasmussen JP, Riviere JE, Monteiro-Riviere NA. Penetration of intact skin by quantum dots with diverse physicochemical properties. Toxicol Sci. 2006;91:159-65.

8. Rouse JG, Yang J, Barron AR, Monteiro-Riviere NA. Fullerenebased amino acid nanoparticle interactions with human epidermal keratinocytes. Toxicol Vitr. 2006;20:1313-20.

9. Gratieri T, Schaefer UF, Jing L, Gao M, Kostka KH, Lopez RFV, et al. Penetration of quantum dot particles through human skin. J Biomed Nanotechnol. 2010;6:586-95.

10. Musazzi UM, Franzè S, Minghetti P, Casiraghi A. Emulsion versus nanoemulsion: how much is the formulative shift critical for a cosmetic product? Drug Deliv Transl Res. 2018;8:414-21.

11. Bachhav YG, Mondon K, Kalia YN, Gurny R, Möller M. Novel micelle formulations to increase cutaneous bioavailability of azole antifungals. J Control Release. 2011;153:126-32.

12. Manca ML, Lai F, Pireddu R, Valenti D, Schlich M, Pini E, et al. Impact of nanosizing on dermal delivery and antioxidant activity of quercetin nanocrystals. J Drug Deliv Sci Technol. 2020;55:101482. 
13. Musazzi UM, Santini B, Selmin F, Marini V, Corsi F, Allevi R, et al. Impact of semi-solid formulations on skin penetration of iron oxide nanoparticles. J Nanobiotechnology. 2017;15:1-10.

14. Musazzi UM, Cencetti C, Franzé S, Zoratto N, Di Meo C, Procacci $\mathrm{P}$, et al. Gellan nanohydrogels: novel nanodelivery systems for cutaneous administration of piroxicam. Mol Pharm. 2018;15:1028-36.

15. Lai F, Caddeo C, Manca ML, Manconi M, Sinico C, Fadda AM. What's new in the field of phospholipid vesicular nanocarriers for skin drug delivery. Int J Pharm. 2020;583:119398.

16. Mezei M, Gulasekharam V. Liposomes - a selective drug delivery system for the topical route of administration I. Lotion dosage form Life Sci Pergamon. 1980;26:1473-7.

17. Mezei M, Gulasekharam V. Liposomes-a selective drug delivery system for the topical route of administration: gel dosage form. J Pharm Pharmacol. 1982;34:473-4.

18. Schreier H, Bouwstra J. Liposomes and niosomes as topical drug carriers: dermal and transdermal drug delivery. J Control Release 1994;1-15.

19. Campani V, Biondi M, Mayol L, Cilurzo F, Franzé S, Pitaro $\mathrm{M}$, et al. Nanocarriers to enhance the accumulation of vitamin K1 into the skin. Pharm Res. 2016;33:893-908.

20. Rocco P, Musazzi UM, Franzè S, Minghetti P. Copies of nonbiological complex drugs: generic, hybrid or biosimilar? Drug Discov Today. 2019;24:250-5.

21. Musazzi UM, Marini V, Casiraghi A, Minghetti P. Is the European regulatory framework sufficient to assure the safety of citizens using health products containing nanomaterials? Drug Discov Today. 2017;22:870-82.

22. Minghetti P, Musazzi UM, Casiraghi A, Rocco P. Old active ingredients in new medicinal products: is the regulatory path coherent with patients' expectations? Drug Discov Today. 2020;25:1337-47.

23. Food and Drug Administration (FDA). Drug products, including biological products, that contain nanomaterials - guidance for industry. 2017 [cited $2021 \mathrm{Jul}$ 27]. Available from: https://www. fda.gov/regulatory-information/search-fda-guidance-documents/ drug-products-including-biological-products-containnanomaterials-guidance-industry.

24. European Medicines Agency (EMA). Reflection paper on surface coating: general issues for consideration regarding parenteral administration of coated nanomedicine products. 2013.

25. European Medicine Agency (EMA). Reflection paper on the data requirements for intravenous liposomal products developed with reference to an innovator liposomal product. 2013.

26. Food and Drug Administration (FDA). Liposome drug products chemistry, manufacturing, and controls; human pharmacokinetics and bioavailability; and labeling documentation guidance for industry. 2018.

27. European Medicines Agency (EMA). Draft Guideline on quality and equivalence of topical products. 2018.

28. Food and Drug Administration (FDA). Transdermal and topical delivery systems - product development and quality considerations. 2019 [cited $2021 \mathrm{Jul}$ 27]. Available from: https://www. fda.gov/regulatory-information/search-fda-guidance-documents/ transdermal-and-topical-delivery-systems-product-developmentand-quality-considerations.

29. Xu X, Khan MA, Burgess DJ. A quality by design (QbD) case study on liposomes containing hydrophilic API: I. Formulation, processing design and risk assessment. Int J Pharm. 2011;419(1-2):52-9.

30. Sabir F, Katona G, Pallagi E, Dobó DG, Akel H, Berkesi D, Kónya Z, Csóka I. Quality-by-design-based development of n-propyl-gallate-loaded hyaluronic-acid-coated liposomes for intranasal administration. Molecules. 2021;26(5):1429.

31. Schlich, M, Palomba, R, Costabile, G, Mizrahy S, Pannuzzo M, Peer D, Decuzzi P. Cytosolic delivery of nucleic acids: the case of ionizable lipid nanoparticles. Bioeng Transl Med. 2021; 6:e10213.

32. Ross K. Towards topical microRNA-directed therapy for epidermal disorders. J Control Release. 2018;269:136-47.

33. Gioria S, Caputo F, Urbán P, Maguire CM, Bremer-Hoffmann S, Prina-Mello A, et al. Are existing standard methods suitable for the evaluation of nanomedicines: some case studies. Nanomedicine. 2018;13:539-54.

34. Maguire CM, Rösslein M, Wick P, Prina-Mello A. Characterisation of particles in solution - a perspective on light scattering and comparative technologies. Sci Technol Adv Mater. 2018;19:732-45.

35. Meller A, Bar-Ziv R, Tlusty T, Moses E, Stavans J, Safran SA. Localized dynamic light scattering: a new approach to dynamic measurements in optical microscopy. Biophys J. 1998;74:1541-8.

36. Lorber B, Fischer F, Bailly M, Roy H, Kern D. Protein analysis by dynamic light scattering: methods and techniques for students. Biochem Mol Biol Educ. 2012;40:372-82.

37. Gilroy EL, Hicks MR, Smith DJ, Rodger A. Viscosity of aqueous DNA solutions determined using dynamic light scattering. Analyst. 2011;136:4159.

38. Naiim M, Boualem A, Ferre C, Jabloun M, Jalocha A, Ravier P. Multiangle dynamic light scattering for the improvement of multimodal particle size distribution measurements. Soft Matter. 2015;11:28-32.

39. Filipe V, Hawe A, Jiskoot W. Critical evaluation of Nanoparticle Tracking Analysis (NTA) by NanoSight for the measurement of nanoparticles and protein aggregates. Pharm Res. 2010;27:796-810.

40. Hawe A, Hulse WL, Jiskoot W, Forbes RT. Taylor dispersion analysis compared to dynamic light scattering for the size analysis of therapeutic peptides and proteins and their aggregates. Pharm Res. 2011;28:2302-10.

41. Amin S, Barnett GV, Pathak JA, Roberts CJ, Sarangapani PS. Protein aggregation, particle formation, characterization and rheology. Curr Opin Colloid Interface Sci. 2014;19:438-49.

42. Mehn D, Caputo F, Rösslein M, Calzolai L, Saint-Antonin F, Courant T, et al. Larger or more? Nanoparticle characterisation methods for recognition of dimers. RSC Adv. 2017;7:27747-54.

43. Hofer C, Göbel R, Deering P, Lehmer A, Breul J. Formulation of interleukin-2 and interferon- $\alpha$ containing ultradeformable carriers for potential transdermal application. Anticancer Res. 1999;19:1505-7.

44. Ntimenou V, Fahr A, Antimisiaris SG. Elastic vesicles for transdermal drug delivery of hydrophilic drugs: a comparison of important physicochemical characteristics of different vesicle types. J Biomed Nanotechnol. 2012;8:613-23.

45. Cosco D, Paolino D, Maiuolo J, Di ML, Carafa M, Ventura CA, et al. Ultradeformable liposomes as multidrug carrier of resveratrol and 5-fluorouracil for their topical delivery. Int J Pharm. 2015;489:1-10.

46. Carrer DC, Vermehren C, Bagatolli LA. Pig skin structure and transdermal delivery of liposomes: a two photon microscopy study. J Control Release. 2008;132:12-20.

47. Cevc G. Lipid vesicles and other colloids as drug carriers on the skin. Adv Drug Deliv Rev. 2004;56:675-711.

48. Opatha SAT, Titapiwatanakun V, Chutoprapat R. Transfersomes: a promising nanoencapsulation technique for transdermal drug delivery. Pharmaceutics. 2020;12:855.

49. Elsayed MMA, Abdallah OY, Naggar VF, Khalafallah NM. Deformable liposomes and ethosomes: mechanism of enhanced skin delivery. Int J Pharm. 2006;322:60-6.

50. Manca ML, Zaru M, Manconi M, Lai F, Valenti D, Sinico C, et al. Glycerosomes: a new tool for effective dermal and transdermal drug delivery. Int J Pharm. 2013;455:66-74.

51. Mura S, Manconi M, Sinico C, Valenti D, Fadda AM. Penetration enhancer-containing vesicles (PEVs) as carriers for cutaneous delivery of minoxidil. Int J Pharm. 2009;380:72-9. 
52. Ogunsola OA, Kraeling ME, Zhong S, Pochan DJ, Bronaugh RL, Raghavan SR. Structural analysis of "flexible" liposome formulations: new insights into the skin-penetrating ability of soft nanostructures. Soft Matter. 2012;8:10226-32.

53. Geusens B, Lambert J, De Smedt SC, Buyens K, Sanders NN, Van Gele M. Ultradeformable cationic liposomes for delivery of small interfering RNA (siRNA) into human primary melanocytes. J Control Release. 2009;133:214-20.

54. Franzé S, Marengo A, Stella B, Minghetti P, Arpicco S, Cilurzo F. Hyaluronan-decorated liposomes as drug delivery systems for cutaneous administration. Int J Pharm. 2018;535:333-9.

55. Van Den Bergh BAI, Wertz PW, Junginger HE, Bouwstra JA. Elasticity of vesicles assessed by electron spin resonance, electron microscopy and extrusion measurements. Int J Pharm. 2001;217:13-24.

56. Franzé S, Donadoni G, Podestà A, Procacci P, Orioli M, Carini $\mathrm{M}$, et al. Tuning the extent and depth of penetration of flexible liposomes in human skin. Mol Pharm. 2017;14:1998-2009.

57. Campani V, Scotti L, Silvestri T, Biondi M, De Rosa G. Skin permeation and thermodynamic features of curcumin-loaded liposomes. J Mater Sci Mater Med. 2020;31.

58. Dendisová M, Jeništová A, Parchaňská-Kokaislová A, Matějka P, Prokopec V, Švecová M. The use of infrared spectroscopic techniques to characterize nanomaterials and nanostructures: a review. Anal Chim Acta. 2018;1031:1-14.

59. Centrone A. Infrared imaging and spectroscopy beyond the diffraction limit. Annu Rev Anal Chem. 2015;8:101-26.

60. Mahmoudi M, Lynch I, Ejtehadi MR, Monopoli MP, Bombelli FB, Laurent S. Protein-nanoparticle interactions: opportunities and challenges. Chem Rev American. 2011;111:5610-37.

61. Guimarães Sá Correia M, Briuglia ML, Niosi F, Lamprou DA. Microfluidic manufacturing of phospholipid nanoparticles: stability, encapsulation efficacy, and drug release. Int J Pharm. 2017;516:91-9.

62. Duangjit S, Opanasopit P, Rojanarata T, Ngawhirunpat T. Characterization and in vitro skin permeation of meloxicam-loaded liposomes versus transfersomes. J Drug Deliv. 2011;2011:1-9.

63. Beasley MM, Bartelink EJ, Taylor L, Miller RM. Comparison of transmission FTIR, ATR, and DRIFT spectra: implications for assessment of bone bioapatite diagenesis. J Archaeol Sci. 2014;46:16-22.

64. Scholtysek P, Achilles A, Hoffmann C-V, Lechner B-D, Meister A, Tschierske C, et al. A T-shaped amphiphilic molecule forms closed vesicles in water and bicelles in mixtures with a membrane lipid. J Phys Chem B. 2012;116:4871-8.

65. Demetzos C. Differential scanning calorimetry (DSC): a tool to study the thermal behavior of lipid bilayers and liposomal stability. J Liposome Res. 2008;18:159-73.

66. Neunert G, Tomaszewska-Gras J, Siejak P, Pietralik Z, Kozak M, Polewski K. Disruptive effect of tocopherol oxalate on DPPC liposome structure: DSC, SAXS, and fluorescence anisotropy studies. Chem Phys Lipids. 2018;216:104-13.

67. Aleskndrany A, Sahin I. The effects of Levothyroxine on the structure and dynamics of DPPC liposome: FTIR and DSC studies. Biochim Biophys Acta - Biomembr. 2020;1862:183245.

68. Korkmaz F, Severcan F. Effect of progesterone on DPPC membrane: evidence for lateral phase separation and inverse action in lipid dynamics. Arch Biochem Biophys. 2005;440:141-7.

69. Severcan F, Kazanci N, Zorlu F. Tamoxifen increases membrane fluidity at high concentrations. Biosci Rep. 2000;20:177-84.

70. Kazanci N, Toyran N, Haris PI, Severcan F. Vitamin D 2 at high and low concentrations exert opposing effects on molecular order and dynamics of dipalmitoyl phosphatidylcholine membranes. Spectroscopy. 2001;15:47-55.

71. Issé BA, Yunes Quartino P, Fidelio GD, Farías RN. Thyroid hormones-membrane interaction: reversible association of hormones with organized phospholipids with changes in fluidity and dipole potential. Chem Phys Lipids. 2013;175-176:131-7.

72. Cater BR, Chapman D, Hawes SM, Saville J. Lipid phase transitions and drug interactions. BBA - Biomembr. 1974;363:54-69.

73. Jain MK, Wu NM. Effect of small molecules on the dipalmitoyl lecithin liposomal bilayer: III. Phase transition in lipid bilayer. J Membr Biol. 1977;34:157-201.

74. Mahrhauser DS, Reznicek G, Gehrig S, Geyer A, Ogris M, Kieweler R, Valenta C. Simultaneous determination of active component and vehicle penetration from F-DPPC liposomes into porcine skin layers. Eur J Pharm Biopharm. 2015;97(Pt A):90-5.

75. Čižinauskas V, Elie N, Brunelle A, Briedis V. Fatty acids penetration into human skin ex vivo: a TOF-SIMS analysis approach. Biointerphases. 2017;12: 011003.

76. Freires IA, Sardi J de CO, de Castro RD, Rosalen PL. Alternative animal and non-animal models for drug discovery and development: bonus or burden?. Pharm Res. 2017;34:681-6.

77. Abd E, Yousuf S, Pastore M, Telaprolu K, Mohammed Y, Namjoshi S, et al. Skin models for the testing of transdermal drugs. Clin Pharmacol Adv Appl. 2016;8:163-76.

78. Godin B, Touitou E. Transdermal skin delivery: predictions for humans from in vivo, ex vivo and animal models 2 . Adv Drug Deliv Rev. 2007;59:1152-61.

79. Takeuchi H, Terasaka S, Sakurai T, Furuya A, Urano H, Sugibayashi K. Variation assessment for in vitro permeabilities through Yucatan micropig skin. Biol Pharm Bull. 2011;34:555-61.

80. Wester RC, Melendres J, Sedik L, Maibach H, Riviere JE. Percutaneous absorption of salicylic acid, theophylline, 2,4dimethylamine, diethyl hexyl phthalic acid, and p-aminobenzoic acid in the isolated perfused porcine skin flap compared to man in vivo. Toxicol Appl Pharmacol. 1998;151:159-65.

81. Cilurzo F, Minghetti P, Sinico C. Newborn pig skin as model membrane in in vitro drug permeation studies: a technical note. AAPS PharmSciTech. 2007;8:97-100.

82. Yamamoto S, Karashima M, Sano N, Fukushi C, Tohyama K, Arai Y, et al. Utility of Göttingen minipigs for prediction of human pharmacokinetic profiles after dermal drug application. Pharm Res. 2017;34:2415-24.

83. Yamamoto S, Sano N, Fukushi C, Arai Y, Karashima M, Hirabayashi $\mathrm{H}$, et al. Utility of hairless rats as a model for predicting transdermal pharmacokinetics in humans. Xenobiotica. 2020;50:831-8.

84. van Ravenzwaay B, Leibold E. A comparison between in vitro rat and human and in vivo rat skin absorption studies. Hum Exp Toxicol. 2004;23:421-30.

85. Takeuchi H, Mano Y, Terasaka S, Sakurai T, Furuya A, Urano $\mathrm{H}$, et al. Usefulness of rat skin as a substitute for human skin in the in vitro skin permeation study. Exp Anim. 2011;60:374-84.

86. Ternullo S, de Weerd L, Flaten GE, Holsæter AM, Škalko-Basnet $\mathrm{N}$. The isolated perfused human skin flap model: a missing link in skin penetration studies? Eur J Pharm Sci. 2017;96:334-41.

87. Ternullo S, de Weerd L, Holsæter AM, Flaten GE, Škalko-Basnet N. Going skin deep: a direct comparison of penetration potential of lipid-based nanovesicles on the isolated perfused human skin flap model. Eur J Pharm Biopharm. 2017;121:14-23.

88. European Medicines Agency (EMA). Guideline on quality of transdermal patches. 2014.

89. Cilurzo F, Musazzi UM, Franzé S, Fedele G, Minghetti P. Design of in vitro skin permeation studies according to the EMA guideline on quality of transdermal patches. Eur J Pharm Sci. 2018;125:86-92.

90. Flaten GE, Palac Z, Engesland A, Filipović-Grčić J, Vanić Ž, Škalko-Basnet N. In vitro skin models as a tool in optimization of drug formulation. Eur J Pharm Sci. 2015;75:10-24.

91. Neupane R, Boddu SHS, Renukuntla J, Babu RJ, Tiwari AK. Alternatives to biological skin in permeation studies: current trends and possibilities. Pharmaceutics. 2020;12:152. 
92. Atrux-Tallau N, Pirot F, Falson F, Roberts MS, Maibach HI. Qualitative and quantitative comparison of heat separated epidermis and dermatomed skin in percutaneous absorption studies. Arch Dermatol Res. 2007;299:507-11.

93. Henning A, Schaefer UF, Neumann D. Potential pitfalls in skin permeation experiments: influence of experimental factors and subsequent data evaluation. Eur J Pharm Biopharm. 2009;72:324-31.

94. Barbero AM, Frasch HF. Pig and guinea pig skin as surrogates for human in vitro penetration studies: a quantitative review. Toxicol Vitr. 2009;23:1-13.

95. Davies DJ, Ward RJ, Heylings JR. Multi-species assessment of electrical resistance as a skin integrity marker for in vitro percutaneous absorption studies. Toxicol In Vitro. 2004;18(3):351-8

96. Supe S, Takudage P. Methods for evaluating penetration of drug into the skin: a review. Ski Res Technol. 2021;27:299-308.

97. Ashtikar M, Nagarsekar K, Fahr A. Transdermal delivery from liposomal formulations - evolution of the technology over the last three decades. J Control Release. 2016;242:126-40.

98. Dragicevic-Curic N, Scheglmann D, Albrecht V, Fahr A. Temoporfin-loaded invasomes: development, characterization and in vitro skin penetration studies. J Control Release. 2008;127:59-69.

99. Dubey V, Mishra D, Dutta T, Nahar M, Saraf DK, Jain NK. Dermal and transdermal delivery of an anti-psoriatic agent via ethanolic liposomes. J Control Release. 2007;123:148-54.

100. Bragagni M, Mennini N, Maestrelli F, Cirri M, Mura P. Comparative study of liposomes, transfersomes and ethosomes as carriers for improving topical delivery of celecoxib. Drug Deliv. 2012;19:354-61.

101. Clares B, Calpena AC, Parra A, Abrego G, Alvarado H, Fangueiro JF, et al. Nanoemulsions (NEs), liposomes (LPs) and solid lipid nanoparticles (SLNs) for retinyl palmitate: effect on skin permeation. Int J Pharm. 2014;473:591-8.

102. Klang V, Schwarz JC, Lenobel B, Nadj M, Auböck J, Wolzt $\mathrm{M}$, et al. In vitro vs. in vivo tape stripping: validation of the porcine ear model and penetration assessment of novel sucrose stearate emulsions. Eur J Pharm Biopharm. 2012;80:604-14.

103. Rukavina Z, Šegvić Klarić M, Filipović-Grčić J, Lovrić J, Vanić Ž. Azithromycin-loaded liposomes for enhanced topical treatment of methicillin-resistant Staphyloccocus aureus (MRSA) infections. Int J Pharm. 2018;553(1-2):109-19.

104. Scognamiglio I, De Stefano D, Campani V, Mayol L, Carnuccio R, Fabbrocini G, et al. Nanocarriers for topical administration of resveratrol: a comparative study. Int J Pharm. 2013;440:179-87.

105. Knudsen NØ, Rønholt S, Salte RD, Jorgensen L, Thormann $\mathrm{T}$, Basse LH, et al. Calcipotriol delivery into the skin with PEGylated liposomes. Eur J Pharm Biopharm. 2012;81:532-9.

106. Muzzalupo R, Tavano L, Cassano R, Trombino S, Ferrarelli T, Picci N. A new approach for the evaluation of niosomes as effective transdermal drug delivery systems. Eur J Pharm Biopharm. 2011;79:28-35

107. Sinico C, Manconi M, Peppi M, Lai F, Valenti D, Fadda AM. Liposomes as carriers for dermal delivery of tretinoin: in vitro evaluation of drug permeation and vesicle-skin interaction. $\mathbf{J}$ Control Release. 2005;103:123-36.

108. Sinico C, Valenti D, Manconi M, Lai F, Fadda AM. Cutaneous delivery of 8-methoxypsoralen from liposomal and niosomal carriers. J Drug Deliv Sci Technol. 2006;16:115-20.

109. Carboni M, Falchi AM, Lampis S, Sinico C, Manca ML, Schmidt $\mathrm{J}$, et al. Physicochemical, cytotoxic, and dermal release features of a novel cationic liposome nanocarrier. Adv Healthc Mater. 2013;2:692-701.
110. Manconi M, Caddeo C, Sinico C, Valenti D, Mostallino MC, Biggio $\mathrm{G}$, et al. Ex vivo skin delivery of diclofenac by transcutol containing liposomes and suggested mechanism of vesicle-skin interaction. Eur J Pharm Biopharm. 2011;78:27-35.

111. Schlich M, Fornasier M, Nieddu M, Sinico C, Murgia S, Rescigno A. 3-hydroxycoumarin loaded vesicles for recombinant human tyrosinase inhibition in topical applications. Colloids Surfaces B Biointerfaces. 2018;171:675-81.

112. Shrotriya SN, Ranpise NS, Vidhate BV. Skin targeting of resveratrol utilizing solid lipid nanoparticle-engrossed gel for chemically induced irritant contact dermatitis. Drug Deliv Transl Res. 2017;7(1):37-52.

113. Ioele G, Tavano L, De Luca M, Ragno G, Picci N, Muzzalupo R. Photostability and ex-vivo permeation studies on diclofenac in topical niosomal formulations. Int J Pharm. 2015;494(1):490-7.

114. Vanti G, Bani D, Salvatici MC, Bergonzi MC, Bilia AR. Development and percutaneous permeation study of escinosomes, escin-based nanovesicles loaded with berberine chloride. Pharmaceutics. 2019;11:682.

115. Balata GF, Faisal MM, Elghamry HA, Sabry SA. Preparation and characterization of ivabradine $\mathrm{HCl}$ transfersomes for enhanced transdermal delivery. Journal of Drug Delivery Science and Technology. 2020;60:101921.

116. Hasan AA, Samir RM, Abu-Zaid SS, Abu Lila AS. Revitalizing the local anesthetic effect of Mebeverine hydrochloride via encapsulation within ethosomal vesicular system. Colloids Surf B Biointerfaces. 2020;194:111208.

117. Niehues H, Bouwstra JA, El Ghalbzouri A, Brandner JM, Zeeuwen PLJM, van den Bogaard EH. 3D skin models for 3R research: the potential of 3D reconstructed skin models to study skin barrier function. Exp Dermatol. 2018;501-11.

118. Bouwstra JA, Helder RWJ, El Ghalbzouri A. Human skin equivalents: impaired barrier function in relation to the lipid and protein properties of the stratum corneum. Adv Drug Deliv Rev. 2021;175:113802.

119. Rheinwald JG, Green H. Formation of a keratinizing epithelium in culture by a cloned cell line derived from a teratoma. Cell. 1975;6:317-30.

120. Sanabria-de la Torre R, Fernández-González A, Quiñones-Vico MI, Montero-Vilchez T, Arias-Santiago S. Bioengineered skin intended as in vitro model for pharmacosmetics, skin disease study and environmental skin impact analysis. Biomedicines 2020;8:464.

121. Tfayli A, Bonnier F, Farhane Z, Libong D, Byrne HJ, BailletGuffroy A. Comparison of structure and organization of cutaneous lipids in a reconstructed skin model and human skin: spectroscopic imaging and chromatographic profiling. Exp Dermatol. 2014;23:441-3.

122. Kennedy AH, Golden GM, Gay CL, Guy RH, Francoeur ML, Mak VHW. Stratum corneum lipids of human epidermal keratinocyte air-liquid cultures: implications for barrier function. Pharm Res. 1996;13:1162-7.

123. Schäfer-Korting M, Bock U, Diembeck W, Düsing H-J, Gamer A, Haltner-Ukomadu E, et al. The use of reconstructed human epidermis for skin absorption testing: results of the validation study. Altern to Lab Anim. 2008;36:161-87.

124. Schmook FP, Meingassner JG, Billich A. Comparison of human skin or epidermis models with human and animal skin in in-vitro percutaneous absorption. Int J Pharm. 2001;215:51-6.

125. Kuntsche J, Bunjes H, Fahr A, Pappinen S, Rönkkö S, Suhonen $\mathrm{M}$, et al. Interaction of lipid nanoparticles with human epidermis and an organotypic cell culture model. Int J Pharm. 2008;354:180-95.

126. Engesland A, Škalko-Basnet N, Flaten GE. Phospholipid vesicle-based permeation assay and EpiSkin ${ }^{\circledR}$ in assessment 
of drug therapies destined for skin administration. J Pharm Sci. 2015;104(3):1119-27.

127. Labouta HI, Thude S, Schneider M. Setup for investigating gold nanoparticle penetration through reconstructed skin and comparison to published human skin data. J Biomed Opt. 2012;18:061218.

128. Pinto F, Fonseca LP, Souza S, Oliva A, de Barros DPC. Topical distribution and efficiency of nanostructured lipid carriers on a 3D reconstructed human epidermis model. J Drug Deliv Sci Technol. 2020;57:101616.

129. Narda M, Ramos-Lopez D, Bustos J, Trullàs C, Granger C. A novel water-based anti-aging suncare formulation provides multifaceted protection and repair against environmental aggressors: evidence from in vitro, ex vivo, and clinical studies. Clin Cosmet Investig Dermatol. 2019;12:533-44.

130. Cadinoiu AN, Rata DM, Atanase LI, Mihai CT, Bacaita SE, Popa M. Formulations based on drug loaded aptamer-conjugated liposomes as a viable strategy for the topical treatment of basal cell carcinoma-in vitro tests. Pharmaceutics. 2021;13(6):866.

131. Zheng D, Giljohann DA, Chen DL, Massich MD, Wang X-Q, Iordanov $\mathrm{H}$, et al. Topical delivery of siRNA-based spherical nucleic acid nanoparticle conjugates for gene regulation. Proc Natl Acad Sci. 2012;109:11975-80.

132. Pivetta TP, Silva LB, Kawakami CM, Araújo MM, Del Lama MPFM, Naal RMZG, et al. Topical formulation of quercetin encapsulated in natural lipid nanocarriers: evaluation of biological properties and phototoxic effect. J Drug Deliv Sci Technol. 2019;53:101148.

133. Wills JW, Hondow N, Thomas AD, Chapman KE, Fish D, Maffeis TG, et al. Genetic toxicity assessment of engineered nanoparticles using a 3D in vitro skin model (EpiDermTM). Part Fibre Toxicol. 2015;13:50.

134. Tyner KM, Wokovich AM, Godar DE, Doub WH, Sadrieh N. The state of nano-sized titanium dioxide ( $\mathrm{TiO} 2)$ may affect sunscreen performance. Int J Cosmet Sci. 2011;33:234-44.

135. Pardeike J, Strohmeier DM, Schrödl N, Voura C, Gruber M, Khinast JG, et al. Nanosuspensions as advanced printing ink for accurate dosing of poorly soluble drugs in personalized medicines. Int J Pharm. 2011;420:93-100.

136. Nakano M, Patel NK. Release, uptake, and permeation behavior of salicylic acid in ointment bases. J Pharm Sci. 1970;59:985-8.
137. Watkinson RM, Guy RH, Hadgraft J, Lane ME. Optimisation of cosolvent concentration for topical drug delivery \&ndash; II: influence of propylene glycol on ibuprofen permeation. Skin Pharmacol Physiol. 2009;22:225-30.

138. Miki R, Ichitsuka Y, Yamada T, Kimura S, Egawa Y, Seki $\mathrm{T}$, et al. Development of a membrane impregnated with a poly(dimethylsiloxane)/poly(ethylene glycol) copolymer for a high-throughput screening of the permeability of drugs, cosmetics, and other chemicals across the human skin. Eur J Pharm Sci. 2015;66:41-9.

139. Touti R, Noun M, Guimberteau F, Lecomte S, Faure C. What is the fate of multi-lamellar liposomes of controlled size, charge and elasticity in artificial and animal skin? Eur J Pharm Biopharm. 2020;151:18-31.

140. Nair RS, Billa N, Leong C-O, Morris AP. An evaluation of tocotrienol ethosomes for transdermal delivery using Strat-M ( membrane and excised human skin. Pharm Dev Technol. 2021;26:243-51.

141. Kuznetsova DA, Vasileva LA, Gaynanova GA, Vasilieva EA, Lenina OA, Nizameev IR, et al. Cationic liposomes mediated transdermal delivery of meloxicam and ketoprofen: optimization of the composition, in vitro and in vivo assessment of efficiency. Int J Pharm. 2021;605:120803.

142. Kansy M, Senner F, Gubernator K. Physicochemical high throughput screening: parallel artificial membrane permeation assay in the description of passive absorption processes. J Med Chem. 1998;41:1007-10.

143. Sinkó B, Garrigues TM, Balogh GT, Nagy ZK, Tsinman O, Avdeef A, et al. Skin-PAMPA: a new method for fast prediction of skin penetration. Eur J Pharm Sci. 2012;45:698-707.

144. Chi C-T, Lee M-H, Weng C-F, Leong MK. In silico prediction of PAMPA effective permeability using a two-QSAR approach. Int J Mol Sci. 2019;20:3170.

145. Casamonti M, Piazzini V, Bilia AR, Bergonzi MC. Evaluation of skin permeability of resveratrol loaded liposomes and nanostructured lipid carriers using a Skin Mimic Artificial Membrane (skin-PAMPA). Drug Deliv Lett. 2019;9:134-45.

Publisher's Note Springer Nature remains neutral with regard to jurisdictional claims in published maps and institutional affiliations. 